\title{
Harmonics for deformed Steenrod operators (Extended Abstract)
}

\author{
François Bergeron 㕧 Adriano Garsia非 and Nolan Wallach 非 \\ ${ }^{1}$ Dépt. de Math., UQAM, Montréal, Québec, H3C 3P8, CANADA. \\ ${ }^{2}$ UCSD, 9500 Gilman Drive \# 0112 La Jolla, CA 92093-0112, USA. \\ received 4 Nov 2009 ,
}

\begin{abstract}
We explore in this paper the spaces of common zeros of several deformations of Steenrod operators. Proofs are omitted in view of pages limitation for the extended abstract.

Résumé. Nous explorons dans cet article l'espace des zéros communs de plusieurs déformations d'opérateurs de Steenrod. Faute de place, les preuves sont omises.
\end{abstract}

Keywords: Harmonic polynomials, Steenrod Operators

\section{Introduction}

In recent years many authors have studied variations on a striking classical result of invariant theory holding for any finite group $W$ of real $n \times n$ matrices generated by reflections. Roughly stated, this result asserts that there is a natural decomposition

$$
\mathbb{R}[\mathbf{x}] \simeq \mathbb{R}[\mathbf{x}]^{W} \otimes \mathbb{R}[\mathbf{x}]_{W}
$$

of the ring of polynomials $\mathbb{R}[\mathbf{x}]$, in $n$ variables $\mathbf{x}=x_{1}, x_{2}, \ldots, x_{n}$, as a tensor product of the ring $\mathbb{R}[\mathbf{x}]^{W}$ of $W$-invariant polynomials, and the " $W$-coinvariant-space" $\mathbb{R}[\mathbf{x}]_{W}$. This last is simply the space obtained as the quotient of the ring $\mathbb{R}[\mathbf{x}]$ by the ideal generated by constant-term-free $W$-invariant polynomials. It is well known that $\mathbb{R}[\mathbf{x}]_{W}$ is isomorphic as a $W$-module to the space $\mathcal{H}_{W}$ of $W$-harmonic polynomials, i.e.: the set of polynomials $f(\mathbf{x})$ that satisfy all partial differential equations of the form $p\left(\partial_{\mathbf{x}}\right) f(\mathbf{x})=0$, where $p\left(\partial_{\mathbf{x}}\right)$ any constant-term-free $W$-invariant polynomial in the partial derivatives $\partial_{i}$.

The purpose of this work is to study twisted versions of this setup. Typically, we replace symmetric operators $\partial_{1}^{k}+\ldots+\partial_{n}^{k}$, by operators of the form

$$
D_{k}:=\sum_{i=1}^{n} a_{i, k} x_{i} \partial_{i}^{k+1}+b_{i, k} \partial_{i}^{k}
$$

\footnotetext{
${ }^{\dagger}$ supported by NSERC-Canada and FQRNT-Québec

‡supported by NSF

$\S$ supported by NSF

1365-8050 @ 2010 Discrete Mathematics and Theoretical Computer Science (DMTCS), Nancy, France
} 
with some parameters $a_{i, k}$ and $b_{i, k}$. We then consider the solution set $\mathcal{H}_{\mathbf{x}}$ of the system of partial differential equations $D_{k} f(\mathbf{x})=0$, for $k \geq 1$. Observe that the operators $D_{k}$ are homogeneous. We say that they are of degree $-k$ since they lower degree of polynomials by $k$. It follows that $\mathcal{H}_{\mathbf{x}}$ is graded by degree. In particular, it makes sense to consider the Hilbert series

$$
H_{n}(t):=\sum_{d \geq 0} t^{d} \operatorname{dim}\left(\pi_{d}\left(\mathcal{H}_{\mathbf{x}}\right)\right)
$$

with $\pi_{d}$ denoting the projection onto the homogeneous component of degree $d$. Clearly the right-hand side of (3) depends on the choice of the parameters $a_{i, k}$ and $b_{i, k}$. Recall that the Hilbert series of the space $\mathcal{H}_{\mathfrak{S}_{n}}$, of $\mathfrak{S}_{n}$-harmonic polynomials (which corresponds to setting $a_{i, k}=0$ and $b_{i, k}=1$ ) is the classical $t$-analog of $n$ !. As we will see later, this is a "generic" value for $H_{n}(t)$.

Before going on with our discussion, let us consider an interesting dual point of view. Following a terminology of Wood [5], we shall say that a polynomial is a hit-polynomial if it can be expressed in the form

$$
f(\mathbf{x})=\sum_{k} D_{k}^{*} g_{k}(\mathbf{x}),
$$

for some polynomials $g_{k}(\mathbf{x})$, with $D_{k}^{*}$ standing for the dual operator of $D_{k}$ with respect to the following scalar product on the ring of polynomials.

For two polynomials $f$ and $g$ in $\mathbb{R}[\mathbf{x}]$, one sets

$$
\langle f, g\rangle:=\left.f\left(\partial_{\mathbf{x}}\right) g(\mathbf{x})\right|_{\mathbf{x}=0} .
$$

In other words, this corresponds to the constant term of the polynomial resulting from the application of the differential operator $f\left(\partial_{\mathbf{x}}\right)$ to $g(\mathbf{x})$. A straightforward computation reveals that, for two monomials $\mathbf{x}^{\mathbf{a}}$ and $\mathbf{x}^{\mathbf{b}}$, we have $\left\langle\mathbf{x}^{\mathbf{a}}, \mathbf{x}^{\mathbf{b}}\right\rangle=\mathbf{a}$ !, if $\mathbf{a}=\mathbf{b}$, and 0 otherwise. Here, as is now almost usual, $\mathbf{a}$ ! stands for $a_{1} ! a_{2} ! \cdots a_{n} !$. This observation makes it clear that 5 indeed defines a scalar product on $\mathbb{R}[\mathbf{x}]$. Moreover, the dual of the operator $\partial_{i}^{k}$ is easily checked to be multiplication by $x_{i}^{k}$. It follows that

$$
D_{k}^{*}=\sum_{i=1}^{n} a_{i, k} x_{i}^{k+1} \partial_{i}+b_{i, k} x_{i}^{k} .
$$

From general basic linear algebra principles, it follows that the space of $\mathcal{H}_{\mathbf{x}}$, of general harmonic polynomials, is orthogonal to the space of hit-polynomials. Moreover, since the subspace of hit-polynomials is homogeneous, the corresponding quotient $\mathbf{C}$ of $\mathbb{R}[\mathbf{x}]$, by this subspace, is isomorphic to $\mathcal{H}_{\mathbf{x}}$ as a graded space.

For, the special case corresponding to setting $a_{i, k}=q$, and $b_{i, k}=1$, for all $i$ and $k$, we denote $\mathcal{H}_{\mathbf{x} ; q}$ resulting space which has been considered by Hivert and Thiéry (in [3]). Using the notation

$$
D_{k ; q}:=\sum_{i=1}^{n} q x_{i} \partial_{i}^{k+1}+\partial_{i}^{k}
$$

Using a simply Lie-bracket calculation, Hivert and Thiéry have observed that $\mathcal{H}_{\mathbf{x} ; q}$ is simply characterized as the common solutions of the two equations $D_{1 ; q} f(\mathbf{x})=0$, and $D_{2 ; q} f(\mathbf{x})=0$. Recall that the ring of polynomials $\mathbb{R}[\mathbf{x}]$ can be considered as a $\mathfrak{S}_{n}$-module for the action that corresponds to permutation of 
the variables. This action restricts to a natural $\mathfrak{S}_{n}$-action on the space $\mathcal{H}_{\mathbf{x} ; q}$, since the operators $D_{k ; q}$ are symmetric. It is classical that $\mathcal{H}_{\mathfrak{S}_{n}}=\mathcal{H}_{\mathbf{x} ; 0}$ is isomorphic, as a $\mathfrak{S}_{n}$-module, to the regular representation of $\mathfrak{S}_{n}$. Hivert-Thiéry go on to state that

Conjecture 1 (Hivert-Thiéry) As $\mathfrak{S}_{n}$-modules, the spaces $\mathcal{H}_{\mathbf{x} ; q}$ is isomorphic to $\mathcal{H}_{\mathfrak{S}_{n}}$, when $q>0$. In particular, this implies that the Hilbert series of $\mathcal{H}_{\mathbf{x} ; q}$ is $[n] !_{t}$.

It follows from 11 that the graded Frobenius characteristic $F_{n}(t)$ of $\mathcal{H}_{\mathbf{x} ; q}\left(\right.$ and $\left.\mathcal{H}_{\mathfrak{S}_{n}}\right)$ is

$$
F_{n}(t)=[n] !_{t}(1-t)^{n} \sum_{\lambda \vdash n} \prod_{k=1}^{n} \frac{1}{d_{k} !}\left(\frac{p_{k}}{k\left(1-t^{k}\right)}\right)^{d_{k}},
$$

where $d_{k}=d_{k}(\lambda)$ is the number of size $k$ parts of $\lambda$.

In this work we generalize and extend the scope of the above conjecture to include the more general operators of (2). Along the way we prove several related results.

\section{Tilde-Harmonics and Hat-Harmonics}

We first consider another interesting special case of (2). Namely, we suppose that all $b_{i, k}$ 's vanish, and all $a_{i, k}$ 's are equal to 1 . Thus, we consider the space of common zeros of the operators $\widetilde{D}_{k}:=\sum_{i=1}^{n} x_{i} \partial_{i}^{k+1}$, which is called the space of tilde-harmonics, and denoted $\widetilde{\mathcal{H}}_{\mathbf{x}}$. We easily check that

$$
\left[\widetilde{D}_{k}, \widetilde{D}_{j}\right]=(k-j) \widetilde{D}_{k+j},
$$

hence $\widetilde{\mathcal{H}}_{\mathbf{x}}$ is simply the set of common zeros of the two equations $\widetilde{D}_{1} f(\mathbf{x})=0$, and $\widetilde{D}_{2} f(\mathbf{x})=0$. The space $\widetilde{\mathcal{H}}_{\mathbf{x}}$ affords a natural action of the symmetric group, and the associated graded Frobenius characteristic is denoted $\widetilde{F}_{n}(t)$. Computer experimentations suggest that the Hilbert series of $\widetilde{\mathcal{H}}_{\mathrm{x}}$ seems to be

$$
\widetilde{\mathcal{H}}_{n}(t)=\sum_{k=0}^{n}\left(\begin{array}{l}
n \\
k
\end{array}\right) t^{k}[k]_{t} !
$$

Modulo a natural conjecture, this follows from a very explicit description of $\widetilde{\mathcal{H}}_{\mathbf{x}}$ outlined below. To state it we need one more family of operators and yet another version of harmonic polynomials. For each $k \geq 1$, consider the operator $\widehat{D}_{k}=\sum_{i=1}^{n} x_{i} \partial_{i}^{k+1}+(k+1) \partial_{i}^{k}$, and introduce the space

$$
\widehat{\mathcal{H}}_{\mathbf{x}}:=\left\{f(\mathbf{x}) \in \mathbb{R}[\mathbf{x}] \mid \widehat{D}_{k} f(\mathbf{x})=0, \quad \forall k \geq 1\right\},
$$

whose elements are said to be "hat-harmonics". We will soon relate the two notions of tilde and hat harmonics. Experimentation suggest that $\widehat{\mathcal{H}}_{\mathbf{x}}$ has dimension $n$ !, and that even more precisely we have the following.

Conjecture 2 As a graded $\mathfrak{S}_{n}$-module, $\widehat{\mathcal{H}}_{\mathbf{x}}$ is isomorphic to the space of $\mathfrak{S}_{n}$-harmonics.

Now, for any given $k$-subset $\mathbf{y}$ of the $n$ variables $\mathbf{x}$, let us consider the space $\widehat{\mathbf{H}}_{\mathbf{y}}$, and write

$$
e_{\mathbf{y}}:=\prod_{x \in \mathbf{y}} x
$$


for the elementary symmetric polynomial of degree $k$ in the variables $\mathbf{y}$. As usual, we define the support of a monomial to be the set of variable that appear in it, with non-zero exponent. Clearly, $\mathbf{y}^{\mathbf{a}}$ has support $\mathbf{y}$ if and only if $\mathbf{y}^{\mathbf{a}}=e_{\mathbf{y}} \mathbf{y}^{\mathbf{b}}$, for some $\mathbf{b}$. Observe that we have the operator identity we can easily check the operator identity

$$
\widetilde{D}_{k} e_{\mathbf{x}}=e_{\mathbf{x}} \widehat{D}_{k}
$$

where $e_{\mathbf{x}}$ stands for the operator of multiplication by $e_{\mathbf{x}}$. We can now state the following remarkable fact.

Theorem 1 The space of tilde-harmonics has the direct sum decomposition

$$
\widetilde{\mathcal{H}}_{\mathbf{x}}=\bigoplus_{\mathbf{y} \subseteq \mathbf{x}} e_{\mathbf{y}} \widehat{\mathcal{H}}_{\mathbf{y}}
$$

if we consider that hat-harmonics for $\mathbf{y}=\emptyset$ are simply the scalars.

The same holds for the more general case of operators $a_{k} \widetilde{D}_{k}$ and $a_{k} \widehat{D}_{k}$, with the $a_{k}$ 's equal to 0 or 1. The intent here is to restrict the set of equations considered to those $k$ for which $a_{k}$ takes the value 1 . The corresponding spaces are denoted $\widetilde{\mathcal{H}}_{\mathrm{x}}^{\mathrm{a}}$ and $\widehat{\mathbf{H}}_{\mathrm{x}}^{\mathrm{a}}$, with similar convention for the corresponding Hilbert series and graded Frobenius characteristics. It follows that, even in this more general context, we have

Corollary 2 For all choices of $a_{k}$,

$$
\widetilde{\mathcal{H}}_{n}^{\mathbf{a}}(t)=\sum_{k=0}^{n}\left(\begin{array}{l}
n \\
k
\end{array}\right) t^{k} \widehat{\mathcal{H}}_{k}^{\mathbf{a}}(t) .
$$

In particular, if conjecture 2 holds then $(8)$ holds. There is an even finer corollary of Theorem 1

Corollary 3 The graded Frobenius characteristic of $\widetilde{\mathcal{H}}_{\mathrm{x}}^{\mathrm{a}}$ is given by the symmetric function

$$
\widetilde{F}_{n}^{\mathbf{a}}(t)=\sum_{k=0}^{n} t^{k} \widehat{F}_{k}^{\mathbf{a}}(t) h_{n-k}(\mathbf{z})
$$

A conjecture of Wood [5, conjecture 7.3] is thus partially addressed in a very explicit manner. Indeed, in view of Theorem 1 . Wood's conjecture is a consequence of Conjecture 2 and the fact that $\widetilde{\mathbf{C}}$ is isomorphic to $\widetilde{\mathcal{H}}_{\mathbf{x}}$ as a graded $\mathfrak{S}_{n}$-module.

\section{More on $q$-harmonics}

We now link the study of harmonics of the $\widetilde{D}_{k}$ to further our understanding of the common zeros of the operators $D_{k: q}$, in the case when $q$ is considered as a formal parameter. Our point of departure is the following important fact. Denote by $\nabla_{k}:=\sum_{i=1}^{n} \partial_{i}^{k}$ the generalized Laplacian, and observe that $D_{k: q}=q \widetilde{D}_{k}+\nabla_{k}$, then we get the following.

Theorem 4 Up to a power of $q$, every $q$-harmonic polynomial $f$ may be written in the form

$$
f=f_{0}+q f_{1}+q^{2} f_{2}+\cdots+q^{m} f_{m}
$$


with $f_{i} \in \mathbb{R}[\mathbf{x}]$, and such that for all $k \geq 1$ we have

$$
\begin{aligned}
& \text { (a) } \nabla_{k} f_{0}=0 \\
& \text { (b) } \nabla_{k} f_{i}=-\widetilde{D}_{k} f_{i-1}, \quad \text { for all } i=2, \ldots, m-1, \\
& \text { (c) } \widetilde{D}_{k} f_{m}=0 .
\end{aligned}
$$

In particular, it follows that for any $r \geq 0$, and any choice of $k_{1}, k_{2}, \ldots, k_{r} \geq 1$, the element

$$
\nabla_{k_{1}} \nabla_{k_{2}} \cdots \nabla_{k_{r}} f_{r}
$$

is a $\mathfrak{S}_{n}$-harmonic polynomial in the usual sense.

Let us now reformulate the expansion of $(13)$ in the form

$$
f=q^{r}\left(f_{0}+q f_{1}+\cdots+q^{m} f_{m}\right) \quad\left(\text { with each } f_{i} \in \mathbb{R}[\mathbf{x}], f_{i} \neq 0\right)
$$

We call $f_{0}$ the first term of $f$ and denote it "FT $(f)$ ". Analogously we say that $f_{m}$ is the last term of $f$ and denote it "LT $(f)$ ". The integer $m$ will be called the length of $f$. We also set

$$
\mathcal{H}_{\mathbf{x}}^{F}:=\mathcal{L}\left[\mathrm{FT}(f) \mid f \in \mathcal{H}_{\mathbf{x} ; q}\right] \quad \text { and } \quad \mathcal{H}_{\mathbf{x}}^{L}:=\mathcal{L}\left[\operatorname{LT}(f) \mid f \in \mathcal{H}_{\mathbf{x} ; q}\right]
$$

to respectively stand for the span of first terms of $q$-harmonics and last terms. Using a theorem of [3] we then get the following remarkable corollary.

Corollary 5 The three spaces $\mathcal{H}_{\mathbf{x}}^{F}, \mathcal{H}_{\mathbf{x}}^{L}$ and $\mathcal{H}_{\mathbf{x} ; q}$ are isomorphic as graded $\mathfrak{S}_{n}$-modules and therefore they are all isomorphic to a submodule of the Harmonics of $\mathfrak{S}_{n}$.

Since the dimension of $\mathcal{H}_{\mathbf{x} ; q}$ is thus bounded above, the single equality $\operatorname{dim} \mathcal{H}_{\mathbf{x} ; q}=n$ ! would imply that $\mathcal{H}_{\mathbf{x} ; q}$ affords the regular representation of $\mathfrak{S}_{n}$. In particular this would yield that $\mathcal{H}_{\mathbf{x}}^{F}$ is none other than the space of harmonics of $\mathfrak{S}_{n}$. Since $\mathcal{H}_{\mathbf{x} ; q}$ is isomorphic to $\mathcal{H}_{\mathbf{x}}^{F}$, as a graded $\mathfrak{S}_{n}$-module, it would follow that $\mathcal{H}_{\mathbf{x} ; q}$ itself is isomorphic to the space of harmonics of $\mathfrak{S}_{n}$ (as a graded $\mathfrak{S}_{n}$-module). Thus the Hivert-Thiéry conjecture would result.

\section{The Kernel of $D_{k}$}

To compute the general space $\mathcal{H}_{\mathrm{x}}$ of harmonic polynomials, we need to find common solutions of the differential equations $D_{k} f(\mathbf{x})=0$, for $k>0$. For each $k$, the kernel of the operator $D_{k}$ may be given a precise explicit description whenever $a_{i, k} d+b_{i, k} \neq 0$, for all $d \in \mathbb{N}$.We lighten the notation by writing simply $a_{i}$ instead of $a_{i, k}$.

The case $k=1$ illustrates all aspects of the method. We construct a set

$$
\left\{\mathbf{y}^{\mathbf{r}}+\Psi_{1}\left(\mathbf{y}^{\mathbf{r}}\right)\right\}_{\mathbf{r} \in \mathbb{N}^{n-1}}
$$

which is a basis of the solution set of $D_{1} f(\mathbf{x})=0$. Here, $\Psi_{1}$ is a linear operator described below. Simply writing $x$ for $x_{n}$, and $\mathbf{y}$ for the set of variables $x_{1}, \ldots, x_{n-1}$, we expand $f \in \mathbb{R}[\mathbf{x}]$ as polynomials in $x$ :

$$
f=\sum_{d} f_{d} \frac{x^{d}}{d !}, \quad \text { with } f_{d} \in \mathbb{R}[\mathbf{y}] .
$$


The effect of $D_{1}$ can then be described in the format

$$
D_{1}\left(\sum_{d} f_{d} \frac{x^{d}}{d !}\right)=\sum_{d}\left[D_{1}\left(f_{d}\right)+\left(d a_{n}+b_{n}\right) f_{d+1}\right] \frac{x^{d}}{d !} .
$$

Setting $a:=a_{n}$ and $b:=b_{n}$, we now assume that $a d+b \neq 0$, for all $d \in \mathbb{N}$. Then, the right-hand side of (19) vanishes if and only we choose $f$ to be such that

$$
f_{d+1}=\frac{-1}{a d+b} D_{1}\left(f_{d}\right)
$$

for all $d \geq 0$. Unfolding this recurrence for the $f_{d}$ 's, we find that every element of the kernel of $D_{1}$ can be written as $f_{0}+\Psi_{1}\left(f_{0}\right)$, if we define the linear operator $\Psi_{1}$ as

$$
\Psi_{1}(g):=\sum_{m \geq 1}(-1)^{m} \frac{D_{1}^{m}(g)}{[a ; b]_{m}} \frac{x^{m}}{m !}, \quad \text { for } g \in \mathbb{R}[\mathbf{y}] .
$$

Here we use the notation $[a ; b]_{m}:=b(a+b)(2 a+b) \cdots((m-1) a+b)$. This leads to the following theorem.

Theorem 6 The collection of polynomials $\mathbf{y}^{\mathbf{r}}+\Psi_{1}\left(\mathbf{y}^{\mathbf{r}}\right)$ is a basis for the kernel of $D_{1}$. In fact, given any polynomial $f$ in the kernel of $D_{1}$, its expansion in terms of this basis is simply obtained as

$$
f=\sum_{\mathbf{r}} a_{\mathbf{r}}\left(\mathbf{y}^{\mathbf{r}}+\Psi_{1}\left(\mathbf{y}^{\mathbf{r}}\right)\right)
$$

with $(f \bmod x)=\sum_{\mathbf{r}} a_{\mathbf{r}} \mathbf{y}^{\mathbf{r}}$.

It follows readily that, whenever $a d+b \neq 0$ for all $d \in \mathbb{N}$, the Hilbert series of the dimension of the kernel of $D_{1}$ is $(1-t)^{1-n}$. In view of Theorem 1 , this implies that the Hilbert series of the kernel of $\widetilde{D}_{1}$ is

$$
1+\sum_{k=1}^{n}\left(\begin{array}{l}
n \\
k
\end{array}\right) t^{k} \frac{1}{(1-t)^{k-1}} .
$$

In fact, we can get an explicit description of this kernel using (9).

We can generalize formula (21) to get a description of the kernel of $D_{k}$ as follows. Observe as before that

$$
D_{k}\left(\sum_{d} f_{d} \frac{x^{d}}{d !}\right)=\sum_{d}\left[D_{k}\left(f_{d}\right)+(a d+b) f_{d+k}\right] \frac{x^{d}}{d !} .
$$

For this expression to be zero, we must have

$$
f_{d+k}=\frac{-1}{a d+b} D_{k}\left(f_{d}\right)
$$

with the same conditions as before on $a$ and $b$. This recurrence has a unique solution given initial values for $f_{d}, 0 \leq d \leq k-1$. Clearly these can be fixed at leisure. Substituting the solution of the recurrence in $f$, we get an element of the kernel of $D_{k}$ if and only if $f$ is of the form

$$
f=\left(f \bmod x^{k}\right)+\Psi_{k}\left(f \bmod x^{k}\right),
$$


with $\Psi_{k}$ the linear operator defined as

$$
\Psi_{k}\left(\sum_{r=0}^{k-1} f_{r} \frac{x^{r}}{r !}\right):=\sum_{m \geq 1} \sum_{r=0}^{k-1}(-1)^{m} \frac{D_{k}^{m}\left(f_{r}\right)}{[a k ; a r+b]_{m}} \frac{x^{k m+r}}{(k m+r) !} .
$$

In particular, it follows that the Hilbert series of the kernel of $D_{k}$ is $\left(1+t+\ldots t^{k-1}\right)(1-t)^{1-n}$.

\section{Some explicit harmonic polynomials}

Common zeros of all $D_{k}$ 's are exactly what we are looking for. Some of these are easy to find when the $D_{k}$ 's are symmetric. Let $\lambda$ be any partition of $n$, and consider a tableau $\tau$ of shape $\lambda$, this is to say a bijection

$$
\tau: \lambda \longrightarrow\{1,2, \ldots, n\},
$$

with $\lambda$ identified with the set of cells of its Ferrers diagram. Recall that, for $\lambda=\lambda_{1} \geq \lambda_{2} \geq \ldots \geq \lambda_{k}>0$, the cells of $\lambda$ are the $n$ pairs $(i, j)$ in $\mathbb{N}^{2}$, such that

$$
1 \leq i \leq \lambda_{j}, \quad 1 \leq j \leq k .
$$

The value $\tau(i, j)$ is called an entry of $\tau$, and it is said to lie in column $i$ of $\tau$. The Garnir polynomial of a $\lambda$-shape tableau $\tau$, is defined to be

$$
\Delta_{\tau}(\mathbf{x}):=\prod_{i, j<k}\left(x_{\tau(i, j)}-x_{\tau(i, k)}\right) .
$$

In other terms, the factors that appear in $\Delta_{\tau}(\mathbf{x})$ are differences of entries of $\tau$ that lie in the same column.

Now, define $\mathcal{V}_{\lambda}$ to be the linear span of the polynomials $\Delta_{\tau}$, for $\tau$ varying in the set of tableaux of shape $\lambda$. In formula,

$$
\mathcal{V}_{\lambda}:=\mathbb{R}\left\{\Delta_{\tau} \mid \tau \text { tableau of shape } \lambda\right\} .
$$

In other words, $\mathcal{V}_{\lambda}$ is the linear span of the $\Delta_{\tau}$. It is well known that this homogeneous (invariant) subspace is an irreducible representation of of $\mathfrak{S}_{n}$ of dimension equal to the number of standard Young tableaux. Moreover, in the ring $\mathbb{R}[\mathbf{x}]$, there exists no isomorphic copy of this irreducible representation lying in some homogeneous component of degree lower then that in which lies $\mathcal{V}_{\lambda}$. It is easy to check that the degrees of all of the $\Delta_{\tau}$ 's, for a tableau of shape $\lambda$, are all equal to

$$
\sum_{i=1}^{\ell(\lambda)}(i-1) \lambda_{i}
$$

which is usually denoted $n(\lambda)$ in the literature (see [4]). This is the smallest possible value for the cocharge of a standard tableau of shape $\lambda$. This fact has the following easy implication.

Proposition 7 For any tableau $\tau$ of shape $\lambda$, the Garnir polynomial $\Delta_{\tau}(\mathbf{x})$ is a zero of $D_{k}$, for $k \geq 1$, whenever $D_{k}$ is symmetric. 
A direct consequence of this is that there is at least one copy of each irreducible representation of $\mathfrak{S}_{n}$ in $\mathcal{H}_{\mathbf{x}}$, when the $D_{k}$ 's are all symmetric. Moreover, under the same conditions, we have

$$
\sum_{\lambda \vdash n} f_{\lambda} t^{n(\lambda)} \ll H_{n}(t)
$$

with “《” denoting coefficient wise inequality, and $H_{n}(t)$ as in 3 .

\section{A new regular sequence and a universal dimension bound}

The goal of this section is to establish a bound for the dimension of $\mathcal{H}_{\mathbf{x} ; q}$ which is valid for all values of $q$. To carry this out we need some auxiliary results from commutative algebra. Let $\mathbb{F}$ be an algebraically closed field and let $\theta_{1}(\mathbf{x}), \theta_{2}(\mathbf{x}), \ldots, \theta_{n}(\mathbf{x})$ be homogeneous polynomials of $\mathbb{F}[\mathbf{x}]$ of respective degrees $d_{1}, d_{2}, \ldots, d_{n}$. The following result is basic.

Proposition 8 The polynomials $\theta_{1}(\mathbf{x}), \theta_{2}(\mathbf{x}), \ldots, \theta_{n}(\mathbf{x})$ form a regular sequence in $\mathbb{F}[\mathbf{x}]$ if and only if the system of equations

$$
\theta_{1}(\mathbf{x})=0, \theta_{2}(\mathbf{x})=0, \ldots, \theta_{n}(\mathbf{x})=0
$$

has, for $\mathbf{x} \in \mathbb{F}^{n}$, the unique solution

$$
x_{1}=0, x_{2}=0, \ldots, x_{n}=0 .
$$

We next make use of this proposition to study the sequence of polynomials

$$
\varphi_{m}(\mathbf{x}):=\sum_{i=1}^{n} a_{i} x_{i}^{m}
$$

for $m \geq 0$. More precisely we seek to obtain conditions on the coefficient sequence

$$
\mathbf{a}=\left(a_{1}, a_{2}, \ldots, a_{n}\right) \in \mathbb{F}^{n}
$$

which assure that, for a given $k \geq 1$, that the polynomials

$$
\varphi_{k}(\mathbf{x}), \varphi_{k+1}(\mathbf{x}), \ldots, \varphi_{k+n-1}(\mathbf{x})
$$

form a regular sequence in $\mathbb{F}[\mathbf{x}]$.

We first observe that the polynomials $\varphi_{m}(\mathbf{x})$, for $m>n$, may be expressed in term of the $\varphi_{k}(\mathbf{x})$ 's, for $1 \leq k \leq n$. Indeed, recall that the ordinary elementary symmetric functions $e_{r}(\mathbf{x})$ may be presented in the form of the identity

$$
\left(t-x_{1}\right)\left(t-x_{2}\right) \cdots\left(t-x_{n}\right)=\sum_{r=0}^{n}(-1)^{r} e_{r}(\mathbf{x}) t^{n-r} .
$$

Setting $t=x_{i}$, we obtain

$$
\sum_{r=0}^{n}(-1)^{r} e_{r}(\mathbf{x}) x_{i}^{n-r}=0
$$


Multiplying both sides by $a_{i} x_{i}^{m-n}$ and isolating $a_{i} x_{i}^{m}$, we get

$$
a_{i} x_{i}^{m}=-\sum_{r=1}^{n}(-1)^{r} e_{r}(\mathbf{x}) a_{i} x_{i}^{m-r} .
$$

Thus, summing up on $i$, the following recurrence results

$$
\varphi_{m}(\mathbf{x})=\sum_{r=1}^{n}(-1)^{r+1} e_{r}(\mathbf{x}) \varphi_{m-r}(\mathbf{x}) .
$$

Unfolding this recurrence, we conclude that $\varphi_{m}$ lies in the ideal $\left(\varphi_{1}, \varphi_{2}, \ldots, \varphi_{n}\right)_{\mathbb{F}[\mathbf{x}]}$, for all $m \geq 1$.

Remark 1 It is interesting to observe that identity 27] yields that

$$
\varphi_{1}(\mathbf{x}), \varphi_{2}(\mathbf{x}), \ldots, \varphi_{n}(\mathbf{x})
$$

is never a regular sequence when $a_{1}+a_{2}+\cdots+a_{n}=0$. Indeed, setting $m=n$ in (27), we get

$$
\varphi_{m}(\mathbf{x})=\sum_{r=1}^{n-1} \varphi_{m-r}(\mathbf{x})(-1)^{r+1} e_{r}(\mathbf{x})+(-1)^{n+1} e_{n}(\mathbf{x})\left(a_{1}+a_{2}+\cdots+a_{n}\right)
$$

and thus the vanishing of $a_{1}+a_{2}+\cdots+a_{n}$ forces $\varphi_{n}(\mathbf{x})$ to vanish modulo the ideal

$$
\left(\varphi_{1}, \varphi_{2}, \ldots, \varphi_{n-1}\right)_{\mathbb{F}[\mathbf{x}]} .
$$

Let us now denote

$$
\Phi_{n}^{k}:=\left(\varphi_{k}, \varphi_{k+1}, \ldots, \varphi_{k+n-1}\right)_{\mathbb{F}[\mathbf{x}]},
$$

the ideal in $\mathbb{F}[\mathbf{x}]$ generated by the $n$ polynomials $\varphi_{\ell}(\mathbf{x})$, with $k \leq \ell \leq k+n-1$. We also write $\Phi_{n}$ for $\Phi_{n}^{1}$. Proposition 8 and 27, combine to yield the following remarkable result.

Theorem 9 For any $k \geq 1$ the sequence

$$
\varphi_{k}(\mathbf{x}), \varphi_{k+1}(\mathbf{x}), \ldots, \varphi_{k+n-1}(\mathbf{x}),
$$

is regular if and only if the sequence

$$
\varphi_{1}(\mathbf{x}), \varphi_{2}(\mathbf{x}), \ldots, \varphi_{n}(\mathbf{x})
$$

is regular.

This given, here and after we need only be concerned with finding conditions on $a_{1}, a_{2}, \ldots, a_{n}$ that assure the regularity of sequence $\varphi_{1}, \varphi_{2}, \ldots, \varphi_{n}$. The following result offers a useful criterion.

Theorem 10 In the ring $\mathbb{F}[\mathbf{x}]$, the polynomials

$$
\varphi_{1}, \varphi_{2}, \ldots, \varphi_{n}
$$


form a regular sequence if and only if we have

$$
x_{i}^{\left(\begin{array}{c}
n \\
2
\end{array}\right)+1} \in \Phi_{n} .
$$

When this happens we have the Hilbert series equalities

$$
F_{\mathbb{F}[x] / \Phi_{n}^{k}}(t)=[k]_{t}[k+1]_{t} \cdots[k+n-1]_{t}
$$

and, in particular,

$$
\operatorname{dim} \mathbb{F}[x] / \Phi_{n}^{k}=(k)(k+1) \cdots(k+n-1) .
$$

Going along the lines of Remark 1, we are now ready to assert the following characterization of the $a_{i}$ 's for which we have regularity.

Theorem 11 For $k>1$, the sequences

$$
\varphi_{k}, \varphi_{k+1}, \ldots, \varphi_{k+n-1}
$$

is regular if and only if we have

$$
a_{i_{1}}+a_{i_{2}}+\cdots+a_{i_{k}} \neq 0,
$$

for all $1 \leq i_{1}<i_{2}<\cdots<i_{k} \leq n$.

We intend to derive the consequences of this assumption in the theory of $q$-harmonics. First, we simply reformulated every statement modulo the substitution of variables

$$
\begin{aligned}
\left(a_{1}, a_{2}, \ldots, a_{n}\right) & \mapsto \mathbf{x}=\left(x_{1}, x_{2}, \ldots, x_{n}\right), \\
\mathbf{x} & \mapsto \xi=\left(\xi_{1}, \xi_{2}, \ldots, \xi_{n}\right),
\end{aligned}
$$

and we now have

$$
\Phi_{n}^{k}=\left(\varphi_{k}(\xi), \varphi_{k+1}(\xi), \ldots, \varphi_{k+n-1}(\xi)\right)_{\mathbb{F}_{\mathbf{x}}[\xi]} .
$$

This given, from Theorem (10) we can derive the following facts about the ring

$$
\mathbb{F}_{\mathbf{x}}\left[\xi_{1}, \xi_{2}, \ldots, \xi_{n}\right],
$$

where now, $\mathbb{F}_{\mathbf{x}}$ denotes the field of rational functions in $\mathbf{x}$ with coefficients in $\mathbb{F}$.

Theorem 12 Let

$$
u_{1}(\xi), u_{2}(\xi), \ldots, u_{(n+1) !}(\xi)
$$

be a monomial basis for the quotient

$$
\mathbb{F}_{\mathbf{x}}[\xi] / \Phi_{n}^{k},
$$

and let $\operatorname{deg}\left(u_{i}\right)=d_{i}$. Then every polynomial $f(\xi) \in \mathbb{F}_{\mathbf{x}}[\xi]$, which is homogeneous of degree $d$, has a unique expansion of the form

$$
f(\xi)=\sum_{i=1}^{(n+1) !} u_{i}(\xi) \sum_{\sum_{k} r_{k}(k+1)=d-d_{i}} a_{i ; \mathbf{r}}(\mathbf{x}) \varphi_{1}^{r_{1}}(\xi), \varphi_{2}^{r_{2}}(\xi) \cdots \varphi_{n}^{r_{n}}(\xi),
$$

where the coefficients $a_{i ; \mathbf{r}}(\mathbf{x})$ are rational functions of $\mathbf{x}$, for $\mathbf{r} \in \mathbb{N}^{n}$. In particular if $d>\left(\begin{array}{c}n+1 \\ 2\end{array}\right)$ then

$$
f(\xi) \equiv 0 \bmod \Phi_{n}^{k}
$$


Let us now denote by $\mathcal{D}(\mathbf{x})$ the algebra of differential operators with coefficients in $\mathbb{F}_{\mathbf{x}}$. Moreover, let $\mathcal{D}_{d}(\mathbf{x})$ denote the subspace of $\mathcal{D}(\mathbf{x})$ consisting of operators of order $d$. More precisely we have $D \in \mathcal{D}_{d}(\mathbf{x})$ if and only if $D$ may be expanded in the form

$$
D=\sum_{|\mathbf{r}| \leq d} a_{\mathbf{r}}(\mathbf{x}) \partial_{\mathbf{x}}^{\mathbf{r}}
$$

with coefficients $a_{\mathbf{r}}(\mathbf{x}) \in \mathbb{F}_{\mathbf{x}}$ such that $a_{\mathbf{r}}(\mathbf{x}) \neq 0$ at least once when $|\mathbf{r}|=d$. We are here extending our vectorial notation to operators, so that

$$
\partial_{\mathbf{x}}^{\mathbf{r}}=\partial_{1}^{r_{1}} \partial_{2}^{r_{2}} \cdots \partial_{n}^{r_{n}}
$$

is an operator of order $|\mathbf{r}|=r_{1}+r_{2}+\ldots+r_{n}$. The degree condition in 37] imply that the polynomial

$$
\sigma(D):=\sum_{|\mathbf{r}|=d} a_{\mathbf{r}}(\mathbf{x}) \xi^{\mathbf{r}}
$$

does not identically vanish. We will refer to $\sigma(D)$ as the "symbol" of $D$.

This given, as a corollary of Theorem [10, we obtain the following basic result for Steenrod operators

Theorem 13 Every operator $D \in \mathcal{D}_{d}(\mathbf{x})$ has an expansion of the form

$$
D=\sum_{i=1}^{(n+1) !} \sum_{\sum_{\ell} r_{k}(k+1) \leq d-d_{i}} a_{i ; \mathbf{r}}(\mathbf{x}) u_{i}\left(\partial_{\mathbf{x}}\right) D_{1 ; q}^{r_{1}} D_{2 ; q}^{r_{2}} \cdots D_{n ; q}^{r_{n}}
$$

where $d_{i}=\operatorname{deg}\left(u_{i}\right)$ and $a_{i ; \mathbf{r}}(\mathbf{x}) \in \mathbb{F}_{\mathbf{x}}$. Note that this holds true for any rational value of $q$.

We may now establish the main goal of this section.

Theorem 14 For any value of $q$ the dimension of the space of $q$-Harmonic polynomials in $\mathrm{x}$ does not exceed $(n+1)$ !

\section{Last Considerations}

Further computer experiments suggest that we have

Conjecture 3 The set $\mathcal{D}_{n}^{\mathrm{a}}$ of common polynomial zeros of the operators

$$
\sum_{i=1}^{n} a_{i} \partial_{x_{i}}^{k} \partial_{y_{i}}^{j}
$$

for all $k, j \in \mathbb{N}$ such that $k+j>0$, is of a bigraded space of dimension $(n+1)^{n-1}$, whenever we have $\mathbf{a}=\left(a_{1}, \ldots, a_{n}\right)$ such that

$$
\sum_{k \in K} a_{k} \neq 0
$$

for all nonempty subsets $K$ of $\{1, \ldots, n\}$. 
Another interesting experimental observation concerning the space of common zeros of $D_{1}$ and $D_{2}$ with general operators

$$
\begin{aligned}
& D_{1}:=\sum_{i=1}^{n} a_{i} x_{i} \partial_{i}^{2}+b_{i} \partial_{i}, \\
& D_{2}:=\sum_{i=1}^{n} c_{i} x_{i} \partial_{i}^{3}+d_{i} \partial_{i}^{2},
\end{aligned}
$$

is that there seem to be conditions, similar to [38), for which this space is always $n$ !-dimensional.

\section{References}

[1] F. Bergeron, N. Bergeron, A. Garsia, M. Haiman et G. Tesler, Lattice Diagram Polynomials and Extended Pieri Rules, Advances in Mathematics, 1999.

[2] M. Haiman, Combinatorics, symmetric functions and Hilbert schemes, In CDM 2002: Current Developments in Mathematics in Honor of Wilfried Schmid \& George Lusztig, International Press Books (2003) 39-112.

[3] F. Hivert and N. Thiéry, Non-commutative deformation of symmetric functions and the integral Steenrod algebra, in: Invariant theory in all characteristics, volume 35 of CRM Proc. Lecture Notes, pages 91-125, Amer. Math. Soc., Providence, RI, 2004.

[4] I. G. Macdonald, Symmetric functions and Hall polynomials, second ed., Oxford Mathematical Monographs, The Clarendon Press Oxford University Press, New York, 1995, With contributions by A. Zelevinsky, Oxford Science Publications.

[5] R.M.W. Wood, Problems in the Steenrod algebra, Bull. London Math. Soc. 30 (1998) 449-517. 